\title{
Nonlinear Differential Equations Modeling the Antarctic Circumpolar Current
}

\author{
Jifeng Chu and Kateryna Marynets (D) \\ Communicated by A. Constantin
}

\begin{abstract}
The aim of this paper is to study one class of nonlinear differential equations, which model the Antarctic circumpolar current. We prove the existence results for such equations related to the geophysical relevant boundary conditions. First, based on the weighted eigenvalues and the theory of topological degree, we study the semilinear case. Secondly, the existence results for the sublinear and superlinear cases are proved by fixed point theorems.
\end{abstract}

Mathematics Subject Classification. 45G99, 58J3, 76B03.

Keywords. Existence, Geophysical flow, Boundary-value problem, Eigenvalues.

\section{Introduction}

The combined forces of gravity and Coriolis (due to Earth's rotation), triggered by the wind stress, drive circulating ocean currents, called gyres, in which the ocean flow adjusts to these two major forces acting on them so that those forces balance one another (see the discussions in [12,13]). Since the Coriolis effect deflects winds, the deflection of gyres is clockwise in the Northern Hemisphere and counter-clockwise in the Southern Hemisphere. These geophysical flows are dominantly horizontal, with horizontal velocities being about a factor $10^{4}$ larger than the vertical velocities [26]. At the same time, friction also plays a role in the generation of the currents (see the discussion in [7]). However, these currents persist after the wind stress has ceased, and in this regime one can neglect frictional effects.

Recently, in [9] a model of the general motion of a gyre in spherical coordinates as a shallow-water flow on a rotating sphere was obtained, in which the gyre motion is described in terms of a stream function by the solution of an elliptic boundary value problem, which can be transfomed into a second-order differential equation by neglecting azimuthal variation. In recent works [2-6], the first author used this equation to model the arctic gyres as a boundary value problem on semi-infinite interval. Such an equation with suitable boundary conditions was also used to model the Antarctic gyres in [8,15,16,18-25]. The presence of a non-uniform current in the Southern Ocean is of great physical relevance, since this region features the largest known waves (see discussions in $[10,27]$ ). Moreover, new flow phenomena appear in wave-current interactions due to non-uniformity in currents (see [11]).

Now let us recall the model of the general motion of a gyre in spherical coordinates. Let $\theta \in[0, \pi)$ be the polar angle (with $\theta=0$ corresponding to the North Pole) and $\varphi \in[0,2 \pi)$ be the angle of longitude (or azimuthal angle) as shown at Fig. 1.

Let $\psi(\theta, \varphi)$ be the stream function and

$$
\Psi(\theta, \varphi)=\psi(\theta, \varphi)+\omega \cos \theta
$$

where $\omega>0$ is the non-dimensional form of the Coriolis parameter. The governing equation for the gyre flow (see the discussion in [9]) is

$$
\frac{1}{\sin ^{2} \theta} \Psi_{\varphi \varphi}+\Psi_{\theta} \cot \theta+\Psi_{\theta \theta}=F(\Psi-\omega \cos \theta)
$$




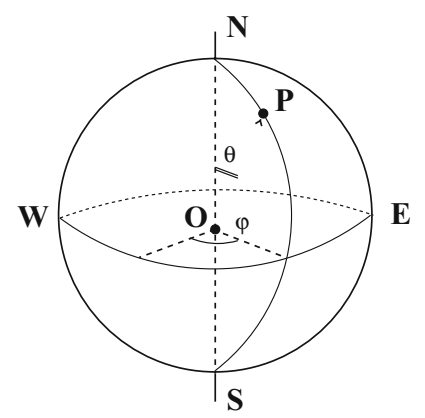

FIG. 1. The azimuthal and polar spherical coordinates $\varphi$ and $\theta$ of a point $P$ on the spherical surface of the Earth: $\theta=0$ and $\theta=\pi$ correspond to the North and South Pole, respectively, while $\theta=\pi / 2$ corresponds to the Equator

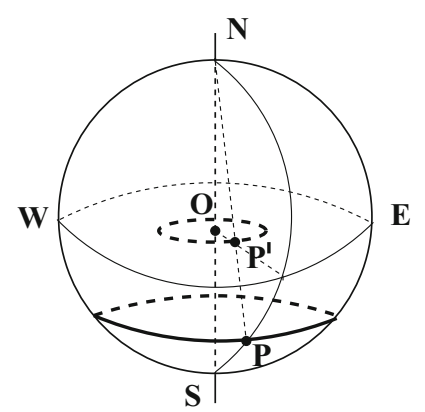

FIG. 2. The stereographic projection $P \mapsto P^{\prime}$ from the North Pole to the equatorial plane: for any point $P$ in the Southern Hemisphere, the straight line connecting it to the North Pole intersects the equatorial plane in a point $P^{\prime}$ belonging to the interior of the circular region delimited by the Equator. The depicted thick band, delimited by two parallels of latitude, represents one of the jets of the Antarctic Circumpolar Current and is mapped bijectively into an annular planar region concentric with the Equator

where $F(\Psi-\omega \cos \theta)$ is the oceanic vorticity, which is typically one order of magnitude larger than the planetary vorticity $2 \omega \cos \theta$, generated by the Earth's rotation. The total vorticity of the gyre flow is the sum of the oceanic vorticity $F(\Psi-\omega \cos \theta)$, and of the planetary vorticity $2 \omega \cos \theta$.

Using the stereographic projection of the unit sphere (centred at the origin) from the North Pole to the equatorial plane (see Fig. 2), Eq. (2) in spherical coordinates

$$
(x, y)=r e^{i \phi} \quad \text { with } \quad r=\cot \left(\frac{\theta}{2}\right)=\frac{\sin \theta}{1-\cos \theta},
$$

where $(r, \phi)$ are the polar coordinates in the equatorial plane, can be transformed into the semilinear elliptic partial differential equation

$$
\Delta \psi+8 \omega \frac{1-\left(x^{2}+y^{2}\right)}{\left(1+x^{2}+y^{2}\right)^{3}}-\frac{4 F(\psi)}{\left(1+x^{2}+y^{2}\right)^{2}}=0,
$$

where $\Delta=\partial_{x}^{2}+\partial_{y}^{2}$ denotes the Laplace operator.

Since the ACC presents a considerable uniformity in the azimuthal direction it corresponds to radially symmetric solutions $\psi=\psi(r)$ of the problem (4). The change of variables

$$
\psi(r)=U(s), \quad s_{1}<s<s_{2},
$$

with $r=e^{-s / 2}$ for

$$
0<s_{1}=-2 \ln \left(r_{+}\right)<s_{2}=-2 \ln \left(r_{-}\right),
$$


and $0<r_{-}<r_{+}<1$, Eq. (4) is transformed to the second-order ordinary differential equation

$$
U^{\prime \prime}(s)-\frac{e^{s}}{\left(1+e^{s}\right)^{2}} F(U(s))+\frac{2 \omega e^{s}\left(1-e^{s}\right)}{\left(1+e^{s}\right)^{3}}=0
$$

for $s_{1}<s<s_{2}$.

The flow in a jet component of the ACC, between the parallels of latitude defined by an appropriate choice of $r_{ \pm} \in(0,1)$ with $r_{+} / r_{-} \in(1,2)$, is modeled by coupling (7) with the boundary conditions

$$
U\left(s_{1}\right)=U\left(s_{2}\right)=0,
$$

which expresses the fact that the boundary of the jet is a streamline - since the flow is steady, this means that a particle there will be confined to the boundary at all times.

Given $0<s_{1}<s_{2}$, the change of variables

$$
u(t)=U(s) \quad \text { with } \quad t=\frac{s-s_{1}}{s_{2}-s_{1}}
$$

transforms (7) and (8) into the equivalent two-point boundary-value problem

$$
\left\{\begin{array}{l}
u^{\prime \prime}=a(t) F(u)+b(t), \quad 0<t<1 \\
u(0)=u(1)=0
\end{array}\right.
$$

where

$$
a(t)=\frac{\left(s_{2}-s_{1}\right)^{2} e^{\left(s_{2}-s_{1}\right) t+s_{1}}}{\left(1+e^{\left(s_{2}-s_{1}\right) t+s_{1}}\right)^{2}}>0,
$$

and

$$
b(t)=-\frac{2 \omega\left(s_{2}-s_{1}\right)^{2} e^{\left(s_{2}-s_{1}\right) t+s_{1}}\left(1-e^{\left(s_{2}-s_{1}\right) t+s_{1}}\right)}{\left(1+e^{\left(s_{2}-s_{1}\right) t+s_{1}}\right)^{3}} .
$$

\section{Semilinear Cases}

In this section, we will establish the existence results for (10) in the semilinear case, that is, the function $F$ is $L^{1}$-Caratheodory and there exist two positive constants $\alpha, \beta$ such that

$$
\alpha \leq \liminf _{|u| \rightarrow \infty} \frac{F(u)}{u} \leq \limsup _{|u| \rightarrow \infty} \frac{F(u)}{u} \leq \beta .
$$

To prove the results, we shall apply the method of topological degree together with some facts on the weighted eigenvalue of the corresponding linear problems. For more details on semilinear differential equations with and without a singularity we refer to [28-30] and the references therein.

Let us first consider the following weighted eigenvalue problem

$$
\begin{aligned}
& u^{\prime \prime}(t)+\lambda a(t) u(t)=0, \quad 0<t<1, \\
& u(0)=u(1)=0,
\end{aligned}
$$

where $a(t)$ is given by (11).

It is easy to show that all eigenvalues $\lambda$ of (13) are positive. Indeed, multiplication (13) by $u$ and integration by parts leads to the inequality

$$
0>-\int_{0}^{1} u^{\prime 2}(s) d s=-\lambda \int_{0}^{1} a(s) u^{2}(s) d s,
$$

from which we conclude that for $\lambda \neq 0$ the Dirichlet spectrum of (13) consists of a sequence of strictly positive numbers, which accumulate at $\infty$.

Additional analysis can be done regarding the Eq. in (13). The Liouville transformation

$$
w(s)=\sqrt[4]{a(t)} u(t)
$$


with

$$
s=\pi \frac{\int_{0}^{t} \sqrt{a(\tau)} d \tau}{\int_{0}^{1} \sqrt{a(\tau)} d \tau}
$$

transforms (13) into the Dirichlet boundary value problem for the Hill's equation:

$$
\left\{\begin{array}{l}
w^{\prime \prime}(t)+[\mu+Q(s)] w(s)=0, \quad 0<s<\pi, \\
w(0)=w(\pi)=0
\end{array}\right.
$$

where

$$
\mu=\frac{\lambda}{\pi^{2}}, Q(s)=\frac{5 a^{\prime 2}(t)}{16 a^{3}(t)}-\frac{a^{\prime \prime}(t)}{4 a^{2}(t)} .
$$

It is a proven fact that the Dirichlet eigenvalues in (15) are simple and can be ordered in an increasing sequence going to infinity (see [17]).

The following result follows essentially from [29, Theorem 2.1].

Theorem 1. The eigenvalue problem (13) has a sequence of eigenvalues:

$$
0<\lambda_{1}(a)<\lambda_{2}(a)<\cdots<\lambda_{k-1}(a)<\lambda_{k}(a)<\cdots<\infty .
$$

Theorem 2. Assume that the function $F(u)$ satisfies the inequality

$$
\limsup _{|u| \rightarrow \infty} \frac{F(u)}{u} \leq \beta,
$$

for some constant $\beta>0$ and

$$
\lambda_{1}(\beta)>1 .
$$

Then the problem (10) has at least one solution.

Proof. Let us deform the Eq. (10) to the following one

$$
u^{\prime \prime}(t)+\beta u(t)=0
$$

and write a homotopic equation in the form:

$$
\left\{\begin{array}{l}
u^{\prime \prime}(t)+G(t, u, \tau), \tau \in[0,1], \\
u(0)=u(1)=0,
\end{array}\right.
$$

where $G(t, u, \tau)=\tau(-a(t) F(u)-b(t))+(1-\tau) \beta u$.

The problem (18) is equivalent to a fixed point equation

$$
u=\mathcal{M}_{\tau}(u), u \in X
$$

in the space

$$
X:=\left\{u:[0, T] \rightarrow \mathbb{R} \text { is } C^{1}[0,1] \text { and } u(0)=u(1)=0\right\}
$$

with the $C^{1}$-norm.

By assumption (17), when $\tau=0$, the problem (18) has only a trivial solution. As the operator $\mathcal{M}_{0}$ is odd in $u \in X$,

$$
\operatorname{deg}\left(\mathcal{M}_{0}, X .0\right)=o d d \neq 0,
$$

which means that the theorem will be proved, if we find a priori bounds for all solutions of (18).

By (16), there exists a constant $0 \leq \psi<+\infty$ such that

$$
u F(u) \leq\left(\beta+\varepsilon_{0}\right)|u|^{2}+\psi,
$$

for all $u \in \mathbb{R}$ for a.e. $t \in[0,1]$. Thus

$$
u(\tau(-a(t) F(u)-b(t))+(1-\tau) \beta u) \leq\left(\beta+\varepsilon_{0}\right)|u|^{2}+\psi,
$$

for all $u \in \mathbb{R}$ for a.e. $t \in[0,1]$ and $\tau \in[0,1]$. 
Let $u(\cdot)$ be the solution of (18) for some $\tau \in[0,1]$. On the one hand, we have

$$
\int_{0}^{1} u u^{\prime \prime} d t=-\int_{0}^{1} u^{\prime 2} d t=-\left\|u^{\prime}\right\|_{2}^{2}
$$

and on the other hand, $\lambda_{1}(a)$ has the following property:

$$
\lambda_{1}(a)=\inf _{u \in W_{2} /\{0\}} \frac{\int_{0}^{1}\left|u^{\prime}\right|^{2} d t}{\int_{0}^{1} a(t)|u|^{2} d t} .
$$

Thus an estimate holds

$$
\begin{aligned}
& \int_{0}^{1} u(\tau(-a(t) F(u)-b(t))+(1-\tau) \beta u) d t \\
& \quad \leq \int_{0}^{1}\left(\beta+\epsilon_{0}\right)|u|^{2}+\psi \\
& \quad \leq\left(\frac{1}{\lambda_{1}(\beta)}+\frac{\epsilon_{0}}{\lambda_{1}(1)}\right)\left\|u^{\prime}\right\|_{2}^{2}+\psi
\end{aligned}
$$

Let us take $\epsilon_{0}<\lambda_{1}(1)\left(1-\frac{1}{\lambda_{1}(a)}\right)$, then

$$
\left\|u^{\prime}\right\|_{2}^{2} \leq \frac{\psi}{1-\frac{1}{\lambda_{1}(\beta)}+\frac{\epsilon_{0}}{\lambda_{1}(1)}}:=M_{0} .
$$

Since $u(0)=u(1)=0$, there exists some constant $c_{1}>0$ such that

$$
\|u\|_{\infty} \leq M_{1} \text {. }
$$

By assumption, the function $F(u)$ is $L^{1}$-Caratheodory function, and it implies that there is some $\Psi \in L^{1}(0,1), \Psi \geq 0$ such that

$$
\mid \tau(-a(t) F(u)-b(t))+(1-\tau) \beta u) \mid \leq \Psi(t),
$$

for all $t, \tau$ and all solutions $u(\cdot)$ of (18).

Due to the Dirichlet boundary conditions in (18), there exists some $t_{1} \in(0,1)$, for which $u^{\prime}\left(t_{1}\right)=0$ and

$$
\begin{aligned}
\left|u^{\prime}(t)\right| & \left.=\mid \int_{t_{1}}^{t}(\tau(-a(s) F(u)-b(s))+(1-\tau) \beta u(s))\right) d s \mid \\
& \leq\|\Psi\|_{1}:=M_{2} .
\end{aligned}
$$

Hence all solutions of the boundary value problem (18) are bounded and the theorem is proved.

Note that condition (17) can be also explicitated variationally (see the considerations in [1]).

Theorem 3. Assume that the function $F(u)$ satisfies condition (12) and there exists an integer $k \geq 2$ such that

$$
\lambda_{k}(\alpha)<1, \quad \lambda_{k-1}(\beta)>1,
$$

then problem (10) has at least one solution.

Proof. Note, that the sequence of eigenvalues $\left\{\lambda_{n}(a)\right\}$ satisfies Theorem 2. Under our assumption, $\beta \geq \alpha$. Therefore, $\lambda(\beta) \leq \lambda(\alpha)$.

Let us take

$$
\alpha<\gamma<\beta
$$

and consider a differential equation

$$
u^{\prime \prime}-\lambda \gamma u=0,
$$

subjected to the boundary constraints (14). 
Since $\gamma>\alpha$, we can conclude that

$$
\lambda_{k-1}(\gamma)<\lambda_{k-1}(\alpha)<1
$$

On the other hand, since $\gamma<\beta$ it follows that

$$
\lambda_{k}(\gamma)>\lambda_{k}(\beta)>1
$$

Thus, for any $n \in \mathbb{N} \lambda_{n}(\gamma) \neq 1$. Hence, the problem (25), (14) has only a trivial solution, and therefore the nonlinear problem (10) has at least one solution.

Remark 1. Moreover, if in Theorems 2 and Theorem 3 function $F(u)$ is assumed to be nondecreasing, it is easy to prove that the solution of the semilinear boundary value problem (10), (14) is unique (see Theorem 5.1 in [25]).

\section{Superlinear Cases}

In this section, we prove one existence result for $(10)$ in the superlinear case at $u=\infty$, which means that

$$
\lim _{u \rightarrow \infty} F(u) / u=\infty .
$$

For example, both

$$
F(u)=u^{\alpha}+\mu u^{\beta}, \quad 0<\alpha<1<\beta, \quad \mu>0,
$$

and

$$
F(u)=e^{\alpha u}, \quad \alpha>0,
$$

are typical superlinear functions. It is easy to see that looking for a solution of (10) is equivalent to looking for a fixed point of the following operator equation

$$
u=\mathcal{F} u,
$$

where

$$
\mathcal{F}(u)(t)=\int_{0}^{1} G(t, s) a(s) F(u(s)) d s+\gamma(t),
$$

where $G(t, s)$ is the Green function given by

$$
G(t, s)= \begin{cases}s(1-t), & \text { if } 0<s<t<1 \\ (1-s) t, & \text { if } 0<t<s<1\end{cases}
$$

and

$$
\gamma(t)=\int_{0}^{1} G(t, s) b(s) d s .
$$

To present the main result, we will use the notations

$$
\mathcal{A}(t)=\int_{0}^{1} G(t, s) \alpha(s) d s,
$$

and

$$
\mathcal{A}^{*}=\max _{t} \mathcal{A}(t), \quad \gamma^{*}=\max _{t} \gamma(t)
$$

Obviously, the functions $\mathcal{A}(t)$ and $\gamma(t)$ are nonnegative, and $\mathcal{A}^{*}>0, \gamma^{*}>0$.

The proof is based on the following nonlinear alternative of Leray-Schauder, which can be found in [14, page 120-130].

Lemma 1. Assume that $\Omega$ is an open subset of a convex set $K$ in a normed linear space $X$ and $p \in \Omega$. Let $T: \bar{\Omega} \rightarrow K$ be a compact and continuous map. Then one of the following two conclusions holds: 
(I) $T$ has at least one fixed point in $\bar{\Omega}$.

(II) There exists $x \in \partial \Omega$ and $0<\lambda<1$ such that

$$
x=\lambda T x+(1-\lambda) p .
$$

Theorem 4. Assume that $F: \mathbb{R} \rightarrow \mathbb{R}$ is continuous and there exists a nondecreasing continuous function $g:[0, \infty) \rightarrow[0, \infty)$ such that

$$
|F(u)| \leq g(|u|), \quad u \in \mathbb{R} .
$$

Suppose further that there exists a positive number $r>\gamma^{*}$ such that

$$
g(r) \mathcal{A}^{*}+\gamma^{*}<r .
$$

Then Eq. (10) has at least one solution $u$ with $0<\|u\|<r$.

Proof. Let us first consider the family of problems

$$
\left\{\begin{array}{l}
u^{\prime \prime}=\lambda a(t) F(u)+b(t), \quad 0<t<1, \\
u(0)=u(1)=0
\end{array}\right.
$$

where $\lambda \in[0,1]$. Note that a solution of (31) is just a fixed point of the operator equation

$$
u=\lambda \mathcal{F} u+(1-\lambda) \gamma(t),
$$

where $\mathcal{F}$ is given as $(28)$.

We claim that for any $\lambda \in[0,1]$, Eq. (32) has no fixed point $x$ with $x \in \partial \Omega$, where

$$
\Omega=\{u \in X:\|u\|<r\},
$$

and $X=C[0,1]$ is a Banach space by taking the norm $\|u\|=\max _{t \in[0,1]}|u(t)|$ for $x \in X$. Obviously, $\Omega$ is an open subset in $X$ with $0 \in \Omega$.

Otherwise, assume that there exists $\lambda_{0} \in[0,1]$ such that Eq. (32) with $\lambda=\lambda_{0}$ has a fixed point $u_{0}$ with $\left\|u_{0}\right\|=r$. Note that

$$
\begin{aligned}
u_{0}(t) & =\lambda_{0} \int_{0}^{1} G(t, s) a(s) F(u(s)) d s+\gamma(t) \\
& \leq \lambda_{0} \int_{0}^{1} G(t, s) a(s) g(|u(s)|) d s+\gamma(t) \\
& \leq g(r) \int_{0}^{1} G(t, s) a(s) d s+\gamma^{*} \\
& \leq g(r) \mathcal{A}^{*}+\gamma^{*}
\end{aligned}
$$

Hence

$$
r \leq g(r) \mathcal{A}^{*}+\gamma^{*}
$$

which is a contradiction to the condition (30).

Obviously $\Omega$ is an open subset in $X$ with $\gamma(t) \in \Omega$ since $\gamma^{*}<r$. Now using Lemma 1, we know that $u=T u$ has a fixed point $u$ in $\Omega$, i.e., Eq. (10) has a solution $u$ with $\|u\|<r$. Finally, one may readily see that $u$ is a nontrivial solution since $u(t) \geq \gamma(t)$.

Example 1. Let $F$ be given as (26). Then

(i) If $\beta<1$, then Eq. (10) has at least one nontrivial solution for each $\mu>0$.

(ii) If $\beta \geq 1$, then Eq. (10) has at least one nontrivial solution for each $0<\mu<\tilde{\mu}$, where $\tilde{\mu}$ is some positive constant. 
Proof. We will apply Theorem 4. To this end, we take

$$
g(x)=x^{\alpha}+\mu x^{\beta}, \quad x>0,
$$

which is non-decreasing in $x \in(0, \infty)$. Now the existence condition (30) becomes

$$
\mu<\frac{r-\mathcal{A}^{*} r^{\alpha}-\gamma^{*}}{\mathcal{A}^{*} r^{\beta}}
$$

for some $r>0$. So Eq. (10) has at least one nontrivial solution for

$$
0<\mu<\tilde{\mu}:=\sup _{r>0} \frac{r-\mathcal{A}^{*} r^{\alpha}-\gamma^{*}}{\mathcal{A}^{*} r^{\beta}} .
$$

Note that $\tilde{\mu}=\infty$ if $\beta<1$ and $\tilde{\mu}<\infty$ if $\beta \geq 1$. We have (i) and (ii).

Acknowledgements. Jifeng Chu was supported by the National Natural Science Foundation of China (Grants Nos. 12071296, 11671118 and 11871273) and by Science and Technology Innovation Plan of Shanghai (Grant No. 20JC1414200). The authors are grateful to reviewers for their valuable comments.

\section{Declarations}

Conflict of interest The authors declare that they have no conflict of interest.

Open Access. This article is licensed under a Creative Commons Attribution 4.0 International License, which permits use, sharing, adaptation, distribution and reproduction in any medium or format, as long as you give appropriate credit to the original author(s) and the source, provide a link to the Creative Commons licence, and indicate if changes were made. The images or other third party material in this article are included in the article's Creative Commons licence, unless indicated otherwise in a credit line to the material. If material is not included in the article's Creative Commons licence and your intended use is not permitted by statutory regulation or exceeds the permitted use, you will need to obtain permission directly from the copyright holder. To view a copy of this licence, visit http://creativecommons.org/licenses/by/4.0/.

Publisher's Note Springer Nature remains neutral with regard to jurisdictional claims in published maps and institutional affiliations.

\section{References}

[1] Buttazzo, G., Giaquinta, M., Hildebrandt, S.: One-Dimensional Variational Problems. An introduction. Oxford University Press, New York (1998)

[2] Chu, J.: On an infinite-interval boundary-value problem in geophysics. Monatsh. Math. 188, 621-628 (2019)

[3] Chu, J.: On a differential equation arising in geophysics. Monatsh. Math. 187, 499-508 (2018)

[4] Chu, J.: On a nonlinear integral equation for the ocean flow in arctic gyres. Q. Appl. Math. 76, 489-498 (2018)

[5] Chu, J.: On a nonlinear model for arctic gyres. Ann. Mat. Pura Appl. (4) 197, 651-659 (2018)

[6] Chu, J.: Monotone solutions of a nonlinear differential equation for geophysical fluid flows. Nonlinear Anal. 166, 144-153 (2018)

[7] Constantin, A.: Frictional effects in wind-driven ocean currents. Geophys. Astrophys. Fluid Dyn. 115, 1-14 (2021)

[8] Constantin, A., Johnson, R.S.: An exact, steady, purely azimuthal flow as a model for the Antarctic Circumpolar Current. J. Phys. Oceanogr. 46, 3585-3594 (2016)

[9] Constantin, A., Johnson, R.S.: Large gyres as a shallow-water asymptotic solution of Euler's equation in spherical coordinates. Proc. R. Soc. A Math. Phys. Eng. Sci. 473(2200), 20170063 (2017)

[10] Constantin, A., Monismith, S.G.: Gerstner waves in the presence of mean currents and rotation. J. Fluid Mech. 820, 511-528 (2017)

[11] Constantin, A., Strauss, W., Varvaruca, E.: Global bifurcation of steady gravity water waves with critical layers. Acta Math. 217, 195-262 (2016)

[12] Gabler, R.E., Petersen, J.F., Trapasso, L.M.: Essentials of Physical Geography. Thomson Brooks/Cole, Belmont (2007)

[13] Garrison, T.: Essentials of Oceanography. National Geographic Society/Cengage Learning, Stamford (2014)

[14] Granas, A., Dugundji, J.: Fixed Point Theory. Springer Monographs in Mathematics. Springer, New York (2003)

[15] Haziot, S.: Study of an elliptic partial differential equation modelling the Antarctic Circumpolar Current. Discrete Contin. Dyn. Syst. 39, 4415-4427 (2019)

[16] Haziot, S., Marynets, K.: Applying the stereographic projection to modeling of the flow of the Antarctic Circumpolar Current. Oceanography 31, 68-75 (2018) 
[17] Magnus, W., Winkler, S.: Hill's Equation. Interscience Publishers, New York (1966)

[18] Martin, C., Quirchmayr, R.: Explicit and exact solutions concerning the Antarctic Circumpolar Current with variable density in spherical coordinates. J. Math. Phys. 60, 101505 (2019)

[19] Marynets, K.: Two-point boundary problem for modeling the jet flow of the Antarctic Circumpolar Current, Electron. J. Differ. Equ., Paper No. 56, 12 pp (2018)

[20] Marynets, K.: A nonlinear two-point boundary-value problem in geophysics. Monatsh. Math. 188, 287-295 (2019)

[21] Marynets, K.: On a two-point boundary-value problem in geophysics. Appl. Anal. 98, 553-560 (2019)

[22] Marynets, K.: On the modeling of the flow of the Antarctic Circumpolar Current. Monatsh. Math. 188, 561-565 (2019)

[23] Marynets, K.: The Antarctic Circumpolar Current as a shallow-water asymptotic solution of Euler's equation in spherical coordinates. Deep Sea Res. Part II Top. Stud. Oceanogr. 160, 58-62 (2019)

[24] Marynets, K.: A weighted Sturm-Liouville problem related to ocean lows. J. Math. Fluid Mech. 20, 929-935 (2018)

[25] Marynets, K.: Study of a nonlinear boundary-value problem of geophysical relevance. Discrete Contin. Dyn. Syst. 39, 4771-4781 (2019)

[26] Viudez, A., Dritschel, D.G.: Vertical velocity in mesoscale geophysical flows. J. Fluid Mech. 483, 199-223 (2015)

[27] Walton, D.W.H.: Antarctica: Global Science from a Frozen Continent. Cambridge University Press, Cambridge (2013)

[28] Yan, P., Zhang, M.: Higher order non-resonance for differential equations with singularities. Math. Methods Appl. Sci. 26, 1067-1074 (2003)

[29] Zhang, M.: Nonuniform nonresonance of semilinear differential equations. J. Differ. Equ. 166, 33-50 (2000)

[30] Zhang, M.: Periodic solutions of equations of Emarkov-Pinney type. Adv. Nonlinear Stud. 6, 57-67 (2006)

Jifeng Chu

Department of Mathematics

Shanghai Normal University

Shanghai 200234

China

e-mail: jifengchu@126.com
Kateryna Marynets

Delft Institute of Applied Mathematics

Delft Univesity of Technology

Mekelweg 4

2628 CD Delft

The Netherlands

e-mail: K.Marynets@tudelft.nl

(accepted: August 10, 2021; published online: August 23, 2021) 